PROCEEDINGS OF THE

AMERICAN MATHEMATICAL SOCIETY

Volume 138, Number 7, July 2010, Pages 2601-2606

S 0002-9939(10)10247-0

Article electronically published on February 26, 2010

\title{
UPPER BOUNDS FOR SERIES INVOLVING MODERATE AND SMALL DEVIATIONS
}

\author{
AUREL SPĂTARU \\ (Communicated by Richard C. Bradley)
}

\begin{abstract}
Let $X, X_{1}, X_{2}, \ldots$ be i.i.d. random variables with $0<E X^{2}=$ $\sigma^{2}<\infty$ and $E X=0$, and set $S_{n}=X_{1}+\cdots+X_{n}$. We prove Paley-type inequalities for series involving probabilities of moderate deviations $P\left(\left|S_{n}\right| \geq\right.$ $\lambda \sqrt{n \log n}), \quad \lambda>0$, and probabilities of small deviations $P\left(\left|S_{n}\right| \geq\right.$ $\lambda \sqrt{n \log \log n}), \lambda>\sigma \sqrt{2}$.
\end{abstract}

\section{INTRODUCTION}

Let $X, X_{1}, X_{2}, \ldots$ be i.i.d. random variables with $0<E X^{2}=\sigma^{2}<\infty$ and $E X=0$, and set $S_{n}=X_{1}+\cdots+X_{n}$. Directed toward obtaining convergence rates for probabilities of moderate deviations $P\left(\left|S_{n}\right| \geq \lambda \sqrt{n \log n}\right), \lambda>0$, and probabilities of small deviations $P\left(\left|S_{n}\right| \geq \lambda \sqrt{n \log \log n}\right), \lambda>\sigma \sqrt{2}$, Davis [3], [2] proved that $S_{1}(\lambda)=\sum_{n \geq 2} \frac{\log n}{n} P\left(\left|S_{n}\right| \geq \lambda \sqrt{n \log n}\right)<\infty, \lambda>0$, and $S_{2}(\lambda)=$ $\sum_{n \geq 2} \frac{1}{n} P\left(\left|S_{n}\right| \geq \lambda \sqrt{n \log \log n}\right)<\infty, \lambda>\sigma \sqrt{2}$. Previously Katz [9] showed that if $p \geq 2$ and $\alpha>1 / 2$, then

$$
\sum_{n \geq 1} n^{p \alpha-2} P\left(\left|S_{n}\right| \geq \lambda n^{\alpha}\right)<\infty, \lambda>0 \Longleftrightarrow E|X|^{p}<\infty .
$$

The case $p=2$ and $\alpha=1$, namely

$$
\sum_{n \geq 1} P\left(\left|S_{n}\right| \geq \lambda n\right)<\infty, \lambda>0 \Longleftrightarrow E X^{2}<\infty
$$

had been settled by Hsu and Robbins [8] and Erdös [4, [5]. Chow and Lai [1] strengthened (1) in the form of a Paley-type inequality by showing that there exists an absolute constant $C>0$, depending only on $p$ and $\alpha$, such that

$$
\sum_{n \geq 1} n^{p \alpha-2} P\left(\left|S_{n}\right| \geq \lambda n^{\alpha}\right) \leq C\left(E|X|^{p}+\left(E X^{2}\right)^{(p \alpha-1) /(2 \alpha-1)}\right), \lambda>0 .
$$

Received by the editors June 7, 2009, and, in revised form, October 9, 2009

2010 Mathematics Subject Classification. Primary 60G50; Secondary 60E15, 60F15.

Key words and phrases. Tail probabilities of sums of i.i.d. random variables, Paley-type inequalities, moderate deviations, small deviations, law of the iterated logarithm.

(C) 2010 American Mathematical Society
Reverts to public domain 28 years from publication 2601 
As for (2), Pruss [13] obtained even sharper bounds; namely, he proved that there are absolute constants $C_{1}, C_{2}>0$ such that

(4)

$$
C_{1} \lambda^{-2} E\left[X^{2} I\{|X| \geq \lambda\}\right] \leq \sum_{n \geq 1} P\left(\left|S_{n}\right| \geq \lambda n\right) \leq C_{2} \lambda^{-2} E\left[X^{2} I\{|X| \geq \lambda\}\right], \lambda>0 .
$$

Motivated by these results and the precise asymptotics $\lim _{\lambda \searrow 0} \lambda^{4} S_{1}(\lambda)=3 \sigma^{4} / 2$ and $\lim _{\lambda \searrow \sigma \sqrt{2}} \sqrt{\lambda^{2}-2 \sigma^{2}} S_{2}(\lambda)=\sigma \sqrt{2}$, due to Gut and Spătaru [6], [7], the purpose of this paper is to get upper bounds similar to (3) for the series $S_{1}(\lambda)$ and $S_{2}(\lambda)$.

Throughout $C$ shall denote absolute positive constants (independent of the distribution of $X$ ), possibly varying from place to place, and $\Phi$ shall stand for the standard normal distribution function.

\section{UPPER BOUND FOR THE SERIES $S_{1}(\lambda)$}

Let $\psi(x)=\sqrt{x \log x}, x \geq 1$, and let $\varphi(x), x \geq 0$, denote the inverse function of $\psi$. Notice that

$$
x^{2}=\varphi(x) \log \varphi(x), x \geq 0 .
$$

For $n \geq 2$, put $X_{n, i}=X_{i} I\left\{\left|X_{i}\right|<\psi(n)\right\}, 1 \leq i \leq n, U_{n}=X_{n, 1}+\cdots+X_{n, n}$ and $\sigma_{n}^{2}=E X_{n, 1}^{2}$. Observe that $\sigma_{n} \nearrow \sigma>0$. It will be clear from the proof of Theorem 1 that we may and do assume that $\sigma_{n}>0$ for all $n \geq 2$. If this assumption fails for $2 \leq n \leq n_{0}$, then $U_{n}=0$ a.s. for $2 \leq n \leq n_{0}$.

Theorem 1. We have

$$
\sum_{n \geq 2} \frac{\log n}{n} P\left(\left|S_{n}\right| \geq \lambda \psi(n)\right) \leq C\left(\lambda^{-2} E X^{2}+\lambda^{-4}\left(E X^{2}\right)^{2}\right), \lambda>0 .
$$

Proof.

Step 1. Suppose first that $X$ is symmetric. Plainly,

$$
\begin{aligned}
P\left(\left|S_{n}\right| \geq \psi(n)\right) & \leq P\left(S_{n} \neq U_{n}\right)+P\left(\left|U_{n}\right| \geq \psi(n)\right) \\
& \leq n P(|X| \geq \psi(n))+P\left(\left|U_{n}\right| \geq \psi(n)\right) .
\end{aligned}
$$

Consequently, we get

$$
\begin{aligned}
& \sum_{n \geq 2} \frac{\log n}{n} P\left(\left|S_{n}\right| \geq \psi(n)\right) \leq \sum_{n \geq 2}(\log n) P(|X| \geq \psi(n)) \\
& \quad+\sum_{n \geq 2} \frac{\log n}{n}\left|P\left(\left|U_{n}\right| \geq \psi(n)\right)-2 \Phi\left(-\frac{\psi(n)}{\sigma_{n} \sqrt{n}}\right)\right|+2 \sum_{n \geq 2} \frac{\log n}{n} \Phi\left(-\frac{\psi(n)}{\sigma_{n} \sqrt{n}}\right) .
\end{aligned}
$$

We have

$$
\begin{aligned}
& \sum_{n \geq 2}(\log n) P(|X| \geq \psi(n))=\sum_{n \geq 2}(\log n) \sum_{k \geq n} P(\psi(k) \leq|X|<\psi(k+1)) \\
& =\sum_{k \geq 2}\left(\sum_{n=2}^{k} \log n\right) P(\psi(k) \leq|X|<\psi(k+1)) \\
& \quad \leq \sum_{k \geq 2} \psi(k)^{2} P\left(\psi(k)^{2} \leq X^{2}<\psi(k+1)^{2}\right) \leq E X^{2} .
\end{aligned}
$$


By the non-uniform estimate of the remainder term in the central limit theorem, due to Nagaev [11] (see also Petrov [12, p. 125]),

$$
\begin{aligned}
\left|P\left(\left|U_{n}\right| \geq \psi(n)\right)-2 \Phi\left(-\frac{\psi(n)}{\sigma_{n} \sqrt{n}}\right)\right| & \leq C \frac{E\left|X_{n, 1}\right|^{3}}{\sqrt{n} \sigma_{n}^{3}}\left(\frac{\sigma_{n} \sqrt{n}}{\psi(n)}\right)^{3} \\
& =C n^{-1 / 2}(\log n)^{-3 / 2} E\left|X_{n, 1}\right|^{3} .
\end{aligned}
$$

Therefore, since $\sum_{n \geq m} n^{-\alpha}(\log n)^{-\beta} \leq C m^{-\alpha+1}(\log m)^{-\beta}, m \geq 2$, whenever $\alpha \geq 1$ and $\beta>0$, applying Fubini's theorem and using (5), we obtain

$$
\begin{aligned}
& \sum_{n \geq 2} \frac{\log n}{n}\left|P\left(\left|U_{n}\right| \geq \psi(n)\right)-2 \Phi\left(-\frac{\psi(n)}{\sigma_{n} \sqrt{n}}\right)\right| \leq C \sum_{n \geq 2} n^{-3 / 2}(\log n)^{-1 / 2} E\left|X_{n, 1}\right|^{3} \\
& \quad=C \sum_{n \geq 2} n^{-3 / 2}(\log n)^{-1 / 2} E\left[|X|^{3} I\{\varphi(|X|)<n\}\right] \\
& \quad=C E\left[|X|^{3} I\{|X|>0\} \sum_{n>\varphi(|X|)} n^{-3 / 2}(\log n)^{-1 / 2}\right] \\
& \quad \leq C E\left[|X|^{3} I\{|X|>0\}(\varphi(|X|) \log \varphi(|X|))^{-1 / 2}\right]=C E X^{2} .
\end{aligned}
$$

As $\sigma_{n} \leq \sigma$ and the function $x \longmapsto \frac{\log x}{x}$ is decreasing for $x \geq e$, we see that

$$
\sum_{n \geq 2} \frac{\log n}{n} \Phi\left(-\frac{\psi(n)}{\sigma_{n} \sqrt{n}}\right) \leq \sum_{n \geq 2} \frac{\log n}{n} \Phi(-\sqrt{\log n} / \sigma) \leq C \int_{1}^{\infty} \frac{\log x}{x} \Phi(-\sqrt{\log x} / \sigma) d x .
$$

By putting $y=\sqrt{\log x} / \sigma$, we then get

$$
\sum_{n \geq 2} \frac{\log n}{n} \Phi\left(-\frac{\psi(n)}{\sigma_{n} \sqrt{n}}\right) \leq C \sigma^{4} \int_{0}^{\infty} 2 y^{3} \Phi(-y) d y=C\left(E X^{2}\right)^{2} .
$$

Now (6) with $\lambda=1$, and so with any $\lambda>0$, follows from (7)-(10).

Step 2. Consider now the general case, and utilize the usual desymmetrization procedure. Let $X^{\prime}, X_{1}^{\prime}, X_{2}^{\prime}, \ldots$ be an independent copy of $X, X_{1}, X_{2}, \ldots$, and put $X^{*}=X-X^{\prime}, X_{n}^{*}=X_{n}-X_{n}^{\prime}$ and $S_{n}^{*}=X_{1}^{*}+\cdots+X_{n}^{*}$. Since $X^{*}, X_{1}^{*}, X_{2}^{*}, \ldots$ are i.i.d. symmetric random variables, on account of Step 1, we have

$$
\sum_{n \geq 2} \frac{\log n}{n} P\left(\left|S_{n}^{*}\right| \geq \lambda \psi(n)\right) \leq C\left(\lambda^{-2} E X^{* 2}+\lambda^{-4}\left(E X^{* 2}\right)^{2}\right), \lambda>0 .
$$

If $\mu\left(S_{n}\right)$ denotes a median of $S_{n}$, then $\left|\mu\left(S_{n}\right)\right|=\left|\mu\left(S_{n}\right)-E S_{n}\right| \leq \sigma \sqrt{2 n}$ (see Loève [10, p. 256]). Hence, by the weak symmetrization inequality (see Loève [10, p. 257]) and (11), we may write

$$
\begin{aligned}
& \sum_{n \geq 2} \frac{\log n}{n} P\left(\left|S_{n}\right| \geq 3 \sigma \psi(n)\right) \leq \sum_{n \geq 2} \frac{\log n}{n} P\left(\left|S_{n}\right| \geq \sigma \psi(n)+\sigma \sqrt{2 n}\right) \\
& \quad \leq \sum_{n \geq 2} \frac{\log n}{n} P\left(\left|S_{n}-\mu\left(S_{n}\right)\right| \geq \sigma \psi(n)\right) \leq 2 \sum_{n \geq 2} \frac{\log n}{n} P\left(\left|S_{n}^{*}\right| \geq \sigma \psi(n)\right) \\
& \quad \leq C\left(\sigma^{-2} E X^{* 2}+\sigma^{-4}\left(E X^{* 2}\right)^{2}\right) .
\end{aligned}
$$


Because $E X^{* 2}=2 E X^{2},(12)$ implies that

$$
\sum_{n \geq 2} \frac{\log n}{n} P\left(\left|S_{n}\right| \geq 3 \lambda \sigma \psi(n)\right) \leq C\left(\lambda^{-2} \sigma^{-2} E X^{2}+\lambda^{-4} \sigma^{-4}\left(E X^{2}\right)^{2}\right), \lambda>0 .
$$

Replacing $3 \lambda \sigma$ by $\lambda$ in (13) leads to (6).

Remark 1. It follows from (6) that $\int_{\lambda_{0}}^{\infty} S_{1}(\lambda) d \lambda<\infty, \lambda_{0}>0$. Thus $E X^{2}<\infty$ and $E X=0$ ensure not only the convergence of the series $\sum_{n \geq 2} \frac{\log n}{n} P\left(\left|S_{n}\right| \geq \lambda \psi(n)\right)$ for any $\lambda>0$, but also the convergence of the double series $\sum_{k \geq 1} \sum_{n \geq 2} \frac{\log n}{n} P\left(\left|S_{n}\right| \geq\right.$ $k \psi(n))$.

Remark 2. On account of (6), we see that $S_{1}(\lambda)=O\left(\lambda^{-2}\right)$ as $\lambda \rightarrow \infty$.

3. UPPER BOUND FOR THE SERIES $S_{2}(\lambda)$

In this section define $\psi(x)=\sqrt{x \log \log x}, x \geq e$, and let $\varphi(x), x \geq 0$, denote the inverse function of $\psi$. Notice that

$$
x^{2}=\varphi(x) \log \varphi(x), x \geq 0 .
$$

For $n \geq e^{e}$, set $X_{n, i}=X_{i} I\left\{\left|X_{i}\right|<\psi(n)\right\}, 1 \leq i \leq n, U_{n}=X_{n, 1}+\cdots+X_{n, n}$ and $\sigma_{n}^{2}=E X_{n, 1}^{2}$. As above we may and do assume that $\sigma_{n}>0$ for all $n \geq e^{e}$.

Theorem 2. We have

(15)

$\sum_{n \geq e^{e}} \frac{1}{n} P\left(\left|S_{n}\right| \geq(\lambda+2 \sigma) \psi(n)\right) \leq C \lambda^{-2} E X^{2}+8 \lambda^{-2} E X^{2}\left(-1+\frac{\lambda}{\sqrt{\lambda^{2}-4 E X^{2}}}\right), \lambda>2 \sigma$.

Proof.

Step 1. Suppose first that $X$ is symmetric. As above, we get

$$
\begin{aligned}
& \sum_{n \geq e^{e}} \frac{1}{n} P\left(\left|S_{n}\right| \geq \psi(n)\right) \leq \sum_{n \geq e^{e}} P(|X| \geq \psi(n)) \\
& \quad+\sum_{n \geq e^{e}} \frac{1}{n}\left|P\left(\left|U_{n}\right| \geq \psi(n)\right)-2 \Phi\left(-\frac{\psi(n)}{\sigma_{n} \sqrt{n}}\right)\right|+2 \sum_{n \geq e^{e}} \frac{1}{n} \Phi\left(-\frac{\psi(n)}{\sigma_{n} \sqrt{n}}\right) .
\end{aligned}
$$

Since $\psi(n) \geq \sqrt{n}$ for $n \geq e^{e}$, we have

$$
\sum_{n \geq e^{e}} P(|X| \geq \psi(n)) \leq \sum_{n \geq e^{e}} P(|X| \geq \sqrt{n}) \leq E X^{2} .
$$

Arguing as in Section 2, we obtain

$$
\left|P\left(\left|U_{n}\right| \geq \psi(n)\right)-2 \Phi\left(-\frac{\psi(n)}{\sigma_{n} \sqrt{n}}\right)\right| \leq C n^{-1 / 2}(\log \log n)^{-3 / 2} E\left|X_{n, 1}\right|^{3} .
$$


Therefore, as $\sum_{n \geq m} \psi(n)^{-3} \leq C m^{-1 / 2}(\log \log m)^{-3 / 2}, m \geq e^{e}$, by Fubini's theorem and (14), we obtain

$$
\begin{aligned}
& \sum_{n \geq e^{e}} \frac{1}{n}\left|P\left(\left|U_{n}\right| \geq \psi(n)\right)-2 \Phi\left(-\frac{\psi(n)}{\sigma_{n} \sqrt{n}}\right)\right| \leq C \sum_{n \geq e^{e}} \psi(n)^{-3} E\left[|X|^{3} I\{\varphi(|X|)<n\}\right] \\
& \quad=C E\left[|X|^{3} \sum_{n>e^{e} \vee \varphi(|X|)} \psi(n)^{-3}\right] \\
& \quad \leq C E\left[|X|^{3}\left(e^{e} \vee \varphi(|X|)\right)^{-1 / 2}\left(\log \log \left(e^{e} \vee \varphi(|X|)\right)\right)^{-3 / 2}\right] \\
& \quad=C E\left[X^{2}(\varphi(|X|) \log \log \varphi(|X|))^{1 / 2}\left(e^{e} \vee \varphi(|X|)\right)^{-1 / 2}\left(\log \log \left(e^{e} \vee \varphi(|X|)\right)\right)^{-3 / 2}\right] \\
& \quad \leq C E X^{2} .
\end{aligned}
$$

For the purpose of the next estimation we assume that $1>\sigma \sqrt{2}$. By putting $y=\sqrt{\log \log x} / \sigma$ and then by partial integration, we have

$$
\begin{aligned}
& 2 \sum_{n \geq e^{e}} \frac{1}{n} \Phi\left(-\frac{\psi(n)}{\sigma_{n} \sqrt{n}}\right) \leq 2 \sum_{n \geq e^{e}} \frac{1}{n} \Phi(-\sqrt{\log \log n} / \sigma) \leq 2 \int_{\left[e^{e}\right]}^{\infty} \frac{1}{x} \Phi(-\sqrt{\log \log x} / \sigma) d x \\
& \leq 4 \sigma^{2} \int_{0}^{\infty} y \Phi(-y) e^{\sigma^{2} y^{2}} d y=4 \sigma^{2}\left(-\frac{1}{2}+\frac{1}{\sqrt{2 \pi}} \int_{0}^{\infty} e^{-\left(1-2 \sigma^{2}\right) y^{2} / 2} d y\right. \\
&=2 \sigma^{2}\left(-1+\frac{1}{\sqrt{1-2 \sigma^{2}}}\right) .
\end{aligned}
$$

From (16)-(19) it follows that

$$
\sum_{n \geq e^{e}} \frac{1}{n} P\left(\left|S_{n}\right| \geq \lambda \psi(n)\right) \leq C \lambda^{-2} E X^{2}+2 \lambda^{-2} E X^{2}\left(-1+\frac{\lambda}{\sqrt{\lambda^{2}-2 E X^{2}}}\right), \lambda>\sigma \sqrt{2} .
$$

Step 2. Consider now the general case, and let $\gamma>2$. By paralleling the reasoning in Step 1 of Section 2 and applying (20), we get

$$
\begin{aligned}
& \sum_{n \geq e^{e}} \frac{1}{n} P\left(\left|S_{n}\right| \geq(\gamma+2) \sigma \psi(n)\right) \\
& \quad \leq C \gamma^{-2} \sigma^{-2} E X^{* 2}+4 \gamma^{-2} \sigma^{-2} E X^{* 2}\left(-1+\frac{\gamma \sigma}{\sqrt{\gamma^{2} \sigma^{2}-2 E X^{* 2}}}\right)
\end{aligned}
$$

where $X^{*}$ is the symmetrized $X$. As $E X^{* 2}=2 E X^{2}$, setting $\gamma \sigma=\lambda$ in (21) leads to $(15)$.

Remark 3. Remarks 1 and 2 of Section 2 have obvious counterparts regarding the series $S_{2}(\lambda)$.

Remark 4. It is reasonable to conjecture that the upper bound in (20) holds true in general, not only for symmetric $X$. The fact that (15) is weaker than (20) is due to the desymmetrization technique employed. 


\section{REFERENCES}

[1] Chow, Y. S. and Lai, T. L. (1978). Paley-type inequalities and convergence rates related to the law of large numbers and extended renewal theory. Z. Wahrscheinlichkeitstheorie verw. Gebiete 45, 1-19. MR507969 (80c:60048)

[2] Davis, J. A. (1968). Convergence rates for the law of the iterated logarithm. Ann. Math. Statist. 39, 1479-1485. MR0253411 (40:6626)

[3] Davis, J. A. (1968). Convergence rates for probabilities of moderate deviations. Ann. Math. Statist. 39, 2016-2028. MR0235599 (38:3903)

[4] Erdős, P. (1949). On a theorem of Hsu and Robbins. Ann. Math. Statist. 20, 286-291. MR0030714 (11:40f)

[5] Erdős, P. (1950). Remark on my paper "On a theorem of Hsu and Robbins". Ann. Math. Statist. 21, 138. MR0032970 (11:375b)

[6] Gut, A. and Spătaru, A. (2000). Precise asymptotics in the Baum-Katz and Davis laws of large numbers. J. Math. Anal. Appl. 248, 233-246. MR.1772594 (2001g:60064)

[7] Gut, A. and Spătaru, A. (2000). Precise asymptotics in the law of the iterated logarithm. Ann. Probab. 28, 1870-1883. MR.1813846 (2001m:60100)

[8] Hsu, P. L. and Robbins, H. (1947). Complete convergence and the law of large numbers. Proc. Nat. Acad. Sci. U.S.A. 33, 25-31. MR0019852 (8:470e)

[9] Katz, M. L. (1963). The probability in the tail of a distribution. Ann. Math. Statist. 34, 312-318. MR0144369 (26:1914)

[10] Loève, M. (1977). Probability Theory. I, 4th edition. Springer-Verlag, Berlin. MR0651017 $(58: 31324 \mathrm{a})$

[11] Nagaev, S. V. (1965). Some limit theorems for large deviations. Theor. Probab. Appl. 10, 214-235. MR0185644 (32:3106)

[12] Petrov, V. V. (1975). Sums of Independent Random Variables. Springer-Verlag, Berlin. MR0388499(52:9335)

[13] Pruss, A. R. (1997). A two-sided estimate in the Hsu-Robbins-Erdős law of large numbers. Stochastic Process. Appl. 70, 173-180. MR.1475661 (99c:60100)

Casa Academiei Romane, Institute of Mathematical Statistics and Applied Mathematics, Calea 13 Septembrie, nr. 13, 76100 Bucharest, Romania 